\section{LEF-1 drives aberrant $\beta$-catenin nuclear localization in myeloid leukemia cells}

\author{
Rhys G. Morgan, ${ }^{1,2}$ Jenna Ridsdale,${ }^{3}$ Megan Payne,${ }^{1}$ Kate J. Heesom, ${ }^{4}$ \\ Marieangela C. Wilson, ${ }^{4}$ Andrew Davidson, ${ }^{1}$ Alexander Greenhough, ${ }^{1}$ Sara \\ Davies, ${ }^{3}$ Ann C. Williams, ${ }^{1}$ Allison Blair,${ }^{1}$ Marian L. Waterman, ${ }^{5}$ Alex Tonks ${ }^{3}$ \\ and Richard L. Darley ${ }^{3}$
}

${ }^{1}$ School of Life Sciences, University of Sussex, Brighton, UK; ${ }^{2}$ School of Cellular and Molecular Medicine, University of Bristol, UK; ${ }^{3}$ Department of Haematology, Division of Cancer and Genetics, School of Medicine, Cardiff University, UK; ${ }^{4}$ University of Bristol Proteomics Facility, UK and ${ }^{5}$ Department of Microbiology and Molecular Genetics, University of California, Irvine, CA, USA

\section{ABSTRACT}

C anonical Wnt/ $\beta$-catenin signaling is frequently dysregulated in myeloid leukemias and is implicated in leukemogenesis. Nuclearlocalized $\beta$-catenin is indicative of active Wnt signaling and is frequently observed in acute myeloid leukemia (AML) patients; however, some patients exhibit little or no nuclear $\beta$-catenin even where cytosolic $\beta$-catenin is abundant. Control of the subcellular localization of $\beta$-catenin therefore represents an additional mechanism regulating Wnt signaling in hematopoietic cells. To investigate the factors mediating the nuclear-localization of $\beta$-catenin, we carried out the first nuclear/cytoplasmic proteomic analysis of the $\beta$-catenin interactome in myeloid leukemia cells and identified putative novel $\beta$-catenin interactors. Comparison of interacting factors between Wnt-responsive cells (high nuclear $\beta$-catenin) versus Wnt-unresponsive cells (low nuclear $\beta$-catenin) suggested the transcriptional partner, LEF-1, could direct the nuclear-localization of $\beta$-catenin. The relative levels of nuclear LEF-1 and $\beta$-catenin were tightly correlated in both cell lines and in primary AML blasts. Furthermore, LEF-1 knockdown perturbed $\beta$ catenin nuclear-localization and transcriptional activation in Wnt-responsive cells. Conversely, LEF-1 overexpression was able to promote both nuclear-localization and $\beta$-catenin-dependent transcriptional responses in previously Wnt-unresponsive cells. This is the first $\beta$-catenin interactome study in hematopoietic cells and reveals LEF-1 as a mediator of nuclear $\beta$ catenin level in human myeloid leukemia.

\section{Introduction}

Canonical Wnt signaling is an evolutionary conserved signal transduction pathway strictly controlled during normal development but frequently dysregulated in cancer. ${ }^{1}$ In the absence of a Wnt ligand, the central mediator of this signaling pathway, $\beta$-catenin, is constitutively phosphorylated by a destruction complex (DC) consisting of GSK3 $\beta$, CK1, Axin and APC, priming it for subsequent degradation by the proteasome. Upon Wnt ligand binding to the Wnt receptors (Frizzled and LRP5/6), the DC becomes saturated with phosphorylated $\beta$-catenin (which cannot be degraded) resulting in cytosolic accumulation of non-phosphorylated $\beta$-catenin. ${ }^{2}$ Following nuclear translocation, $\beta$-catenin complexes with the T-cell factor (TCF)/lymphoid enhancer factor (LEF) transcriptional regulators and promotes activation of proto-oncogenic Wnt target genes, like c-myc, cyclinD1 and survivin (http://web.stanford.edu/group/nusselab/cgi-bin/wnt/target_genes). Thus, Wnt signaling activation is dependent on the movement of $\beta$-catenin into the nucleus, yet this remains a poorly understood process in blood cells. $\beta$-Catenin lacks canonical nuclear-localization or -export sequences and its subcellular distribution has instead been associated with multiple factors in context-dependent settings. ${ }^{3}$

$\beta$-Catenin is frequently over-expressed in acute myeloid leukemia (AML) ${ }^{4}$ where its expression correlates with inferior patient survival. ${ }^{5} \beta$-Catenin has been shown
Ferrata Storti Foundation
Haematologica 2018
Volume 104(7):1365-1377

\section{Correspondence:}

RICHARD DARLEY

darley@cardiff.ac.uk

RHYS MORGAN

rhys.morgan@sussex.ac.uk

Received: July 26, 2018.

Accepted: January 3, 2019.

Pre-published: January 10, 2019.

doi:10.3324/haematol.2018.202846

Check the online version for the most updated information on this article, online supplements, and information on authorship \& disclosures: www.haematologica.org/content/104/7/1365

(C)2019 Ferrata Storti Foundation

Material published in Haematologica is covered by copyright. All rights are reserved to the Ferrata Storti Foundation. Use of published material is allowed under the following terms and conditions:

https://creativecommons.org/licenses/by-nc/4.0/legalcode. Copies of published material are allowed for personal or internal use. Sharing published material for non-commercial purposes is subject to the following conditions:

https://creativecommons.org/licenses/by-nc/4.0/legalcode, sect. 3. Reproducing and sharing published material for commercial purposes is not allowed without permission in writing from the publisher. 
to play a key role in the initiation of AML and chronic myeloid leukemia (CML). ${ }^{6,7}$ Furthermore, frequent chromosomal aberrations driving AML and CML are known to co-operate with $\beta$-catenin., ${ }^{8,9}$ Key to the activation of Wnt signaling is the movement of $\beta$-catenin into the nucleus and this is frequently observed in AML. ${ }^{10}$ We have previously demonstrated that approximately $10 \%$ of primary AML patient blast samples exhibit little nuclear $\beta$-catenin expression, despite substantial cytosolic levels, a phenomenon replicated in $10-20 \%$ of myeloid leukemia cell lines upon Wnt stimulation. ${ }^{3,11}$ In fact, this is characteristic of normal human hematopoietic stem/progenitor cells (HSPC) which similarly limit $\beta$-catenin nuclear-localization, possibly to protect normal HSC from detrimental levels of Wnt signaling. ${ }^{12}$ The permissive nuclear-localization of $\beta$-catenin observed in myeloid leukemias is therefore aberrant and warrants further investigation.

To better understand $\beta$-catenin nuclear-localization mechanisms in myeloid leukemia cells, we generated the first $\beta$-catenin interactomes in hematopoietic cells. These analyses have shown that LEF-1, a $\beta$-catenin-dependent transcription factor, can also regulate the level of nuclear $\beta$-catenin in myeloid leukemia cells. The relative level of nuclear LEF-1 expression correlates with relative nuclear levels of $\beta$-catenin in primary AML patient blasts indicating this axis has clinical relevance. Furthermore, the nuclear-localization of $\beta$-catenin can be promoted by LEF-1 overexpression and conversely is reduced by LEF-1 knockdown. Finally, we demonstrate LEF-1 expression is suppressed in Wnt-unresponsive cells through rapid proteolytic degradation that is not observed in Wnt-responsive cells. Overall, this study characterizes $\beta$-catenin interactions within a hematopoietic context and identifies LEF-1 as a regulator of nuclear $\beta$-catenin localization in human leukemia.

\section{Methods}

\section{Patient samples, cell culture and $\beta$-catenin \\ stabilization}

Bone marrow, peripheral blood or leukapheresis samples from patients diagnosed with AML/myelodysplastic syndromes (MDS) (for clinical information see Online Supplementary Table S1) were collected in accordance with the Declaration of Helsinki and with approval of University Hospitals Bristol NHS Trust and London Brent Research Ethics Committee. Mononuclear cells were separated using Ficoll-Hypaque (Sigma-Aldrich, Poole, UK) and samples with $\geq 80 \%$ viability included in the study. K562, HEL, ML-1, U937, THP1 and PLB-985 cell lines (ECACC, Salisbury, UK) were cultured as previously described. ${ }^{11}$ For proliferation assays, cell lines were seeded in triplicate at $1 \times 10^{5} / \mathrm{mL}$ into 24 -well plates within medium containing 10, 5, 1 or $0.5 \%$ fetal bovine serum (Labtech, East Sussex, UK) and cellular density counted using a hemocytometer at 24, 48 and 72 hours (h). For Wnt signaling activation, cell lines were treated with $5 \mu \mathrm{M}$ of the GSK-3 $\beta$ inhibitor CHIR99021 (Sigma-Aldrich) or $1 \mu \mathrm{g} / \mathrm{mL}$ recombinant murine Wnt3a (Peprotech, London, UK) for $16 \mathrm{~h}$ (unless otherwise stated) at $37^{\circ} \mathrm{C}$.

\section{Nuclear/cytoplasmic fractionation}

$2-8 \times 10^{6}$ cells were washed in PBS and resuspended in $250 \mu \mathrm{L}$ cytoplasmic lysis buffer (10 mM Tris- $\mathrm{HCl}$ (pH8), $10 \mathrm{mM} \mathrm{NaCl}, 1.5$ $\mathrm{mM} \mathrm{MgCl2}$, 0.5\% Igepal-CA630/NP40) containing complete ${ }^{\mathrm{TM}}$
Mini Protease-Inhibitor Cocktail (PIC; Sigma-Aldrich) for $10 \mathrm{~min}$ utes $(\mathrm{min})$ at $4^{\circ} \mathrm{C}$. The supernatant (cytosolic fraction) was recovered following centrifugation at $800 \mathrm{~g}$ for $5 \mathrm{~min}$, and the nuclear pellet washed twice with PBS. Nuclear pellets were resuspended in lysis buffer (Cell Signaling Technology, Leiden, the Netherlands) containing PIC and incubated for $45 \mathrm{~min}$ with sonication to maximize nuclear lysis. Insoluble material was removed at $21,000 \mathrm{~g}$ for $10 \mathrm{~min}$ and solubilized nuclear fractions stored at $-80^{\circ} \mathrm{C}$.

\section{Lentiviral transduction}

K562 and HEL cells were lentivirally-transduced with the $\beta$-catenin-activated reporter (BAR) or mutant 'found unresponsive' control (fuBAR) system as previously. ${ }^{11}$ For LEF-1 knockdown/overexpression, cells were lentivirally-transduced with human LEF-1 shRNA (TRCN0000-020163, -413476, -418104, -428178 and -428355 , MISSION® Sigma), or LEF-1 overexpression vector (pLV-EGFP:T2A:Puro-EF1A>hLEF-1 VectorBuilder, NeuIsenburg, Germany). Cells transduced with scrambled shRNA/empty vector served as controls.

\section{$\beta$-catenin co-immunoprecipitation and immunoblotting}

For co-immunoprecipitation (co-IP), $8 \mu \mathrm{g}$ of crosslinked $\beta$-catenin (Clone-14) or IgG (Clone MOPC-31C) antibody (Becton Dickinson, Oxford, UK) were incubated with $1 \mathrm{mg}$ of either precleared cytoplasmic or nuclear lysate overnight at $4^{\circ} \mathrm{C}$ (Online Supplementary Methods). Subsequently beads were washed five times prior to proteomic analyses or boiled for $95^{\circ} \mathrm{C}$ for $5 \mathrm{~min}$ following washes for immunoblotting. Immunoblotting was performed as previously described ${ }^{13}$ using antibodies to total $\beta$-Catenin (as above), phosphorylated $\beta$-catenin (Ser33/37/Thr41), $\beta$-Catenin (Clone-5), E-Cadherin (Clone-36), GSK3 $\beta$ (Clone-7; $\mathrm{BD}), \alpha$-tubulin (DM1A), lamin A/C (4C11; Sigma), Axin1 (C76H11), Axin2 (76G6), TCF-4 (C48H11), LEF-1 (C12A5), Survivin (71G4B7), CyclinD1 (92G2; Cell Signaling Technology), active $\beta$-catenin (8E7, Merck-Millipore, Watford, UK) and c-MYC (9E10; Santa Cruz, Heidelberg, Germany). $\beta$-Catenin and LEF-1 densitometry were performed as described in the Online Supplementary Methods.

\section{Mass spectrometry and data analyses}

Cytosolic or nuclear $\beta$-Catenin/IgG co-IPs were prepared and analyzed by mass spectrometry (MS) as detailed in the Online Supplementary Methods. Post-acquisition, duplicate values and proteins detected by only a single peptide were first removed. Tandem Mass Tag (TMT) ratios were imported into Perseus v.1.5.6.0 (Max Planck Institute of Biochemistry, Munich, Germany) followed by logarithmic transformation, normalization (through median subtraction) and removal of proteins not present in at least two of three replicates. A one-sample $t$-test was performed with significance of protein binding (- $\log _{10} P$-value) plotted versus fold change in protein binding $\left(\log _{2}\right)$. The MS proteomics data have been deposited with the ProteomeXchange Consortium (http://proteomecentral.proteomexchange.org) via the PRIDE partner repository with the dataset identifier PXD009305. Interaction specificity was assessed using the publicly available CRAPome database (Contaminant Repository for Affinity Purification: http://Www. crapome.org).

\section{Assessment of T-cell factor reporter and flow cytometry}

Activity of BAR lentiviral construct was performed as previously described. ${ }^{11}$ Multi-parameter flow cytometric measurements were acquired using a MACSQuant ${ }^{\circledR}$ Analyzer 10 in conjunction with MACSQuantify ${ }^{\text {TM }}$ v.2.8 (Miltenyi Biotec, Bisley, UK) or an Accuri C6 in conjunction with C sampler software v.1.0.264.21 (BD). Post-acquisition analyses were performed using FlowJo 
v.10.5.3 (Tree Star Inc., Ashland, OR, USA). Threshold for TCF reporter fluorescence was set using matched-controls expressing mutant 'found unresponsive' fuBAR. Cell viability was assessed using $2 \mu \mathrm{g} / \mathrm{mL}$ propidium iodide (Miltenyi Biotech).

\section{Statistical analysis}

Statistical analyses were performed using GraphPad Prism v.7.0 (GraphPad Software Inc., San Diego, CA, USA) and Perseus. Correlation was assessed using a Spearman Rank correlation coefficient (R). Significance of difference was assessed using a onesample or Student $t$-test and data represents mean \pm one Standard Deviation (SD) derived from three biological replicates.

\section{Results}

Myeloid leukemia cell lines exhibit heterogeneous nuclear $\beta$-catenin localization and Wnt activation

Previously we showed that myeloid leukemia cell lines vary markedly in their capacity for nuclear $\beta$-catenin local- ization upon Wnt stimulation, mimicking the heterogeneity of nuclear $\beta$-catenin translocation in AML patients. ${ }^{11}$ To investigate the mechanistic basis for this, we selected two sets of myeloid leukemia lines which differed markedly in Wnt signaling output in response to agonist. K562 and HEL were Wnt-responsive cell lines that localized high levels of $\beta$-catenin into the nucleus upon treatment with the Wnt agonist, CHIR99021, a GSK3 $\beta$ inhibitor (Figure 1A). Cell viability was not significantly affected by $16 \mathrm{~h}$ CHIR99021 treatment (Online Supplementary Figure S1A and $B$ ). Similar patterns of subcellular localization were observed for the active (non-phosphorylated) form of $\beta$ catenin in response to CHIR99021 (Online Supplementary Figure S1C). As expected, the phosphorylated forms of $\beta$ catenin (Ser33/37/Thr41) were reduced upon CHIR99021 treatment (Online Supplementary Figure S1C). Correspondingly, these lines showed robust induction of a TCF reporter (a measure of $\beta$-catenin-dependent transcription), whilst cells expressing reporter with a mutated TCF

A

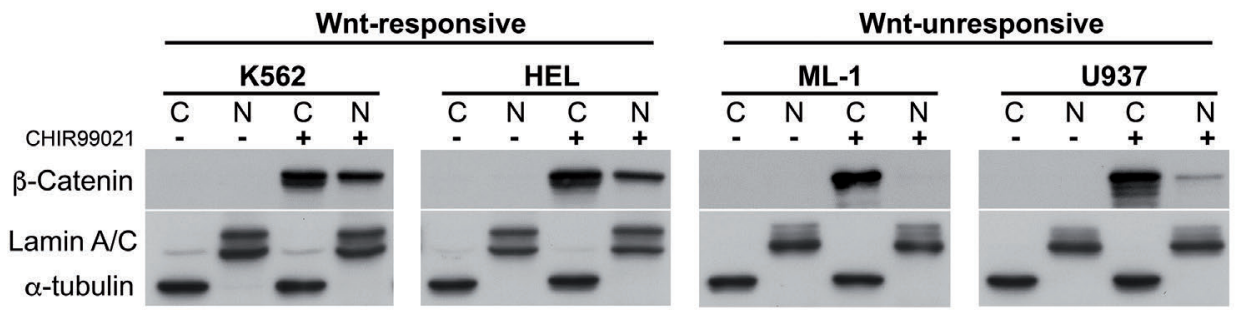

B

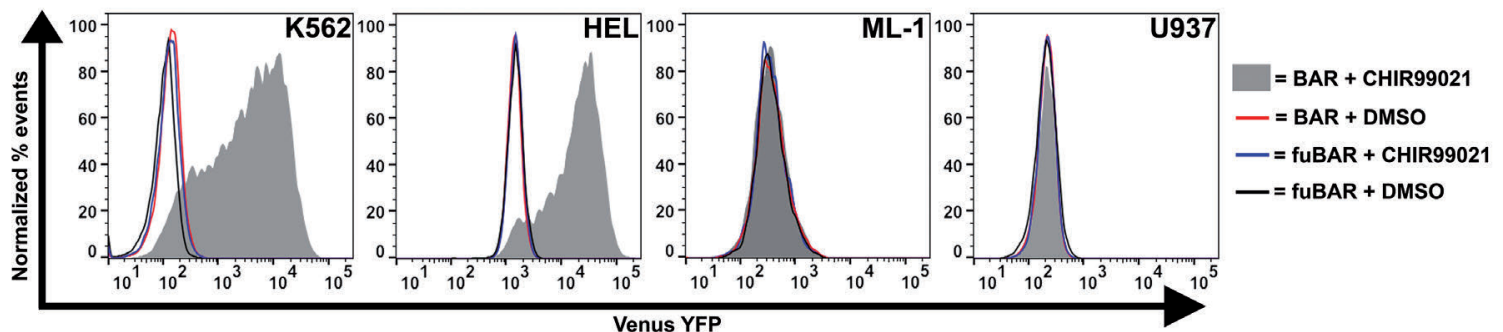

C

D
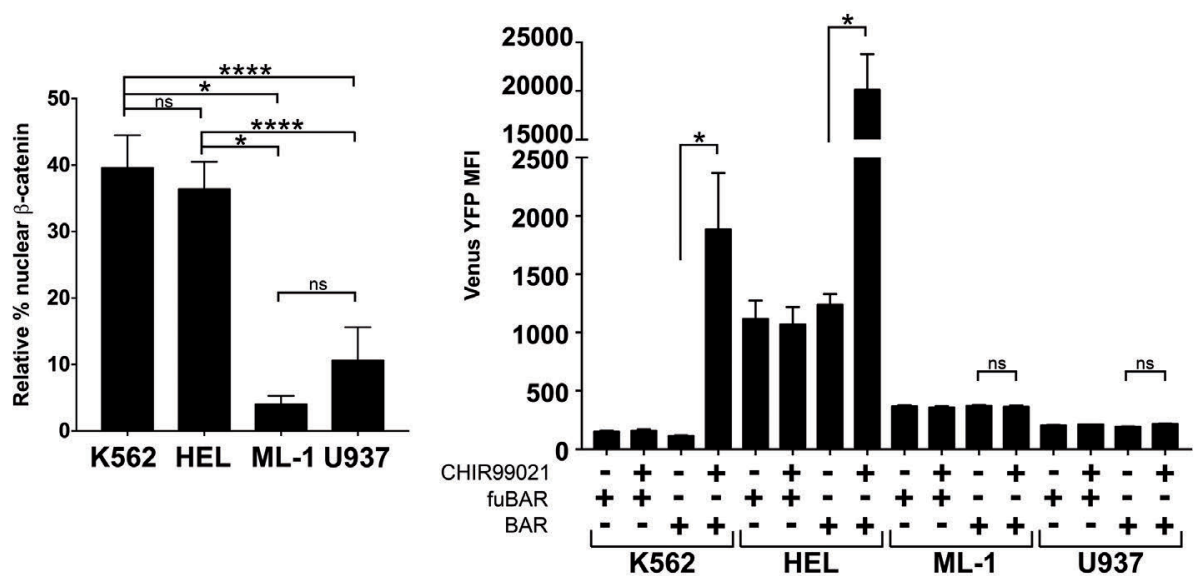

Figure 1. Myeloid leukemia cell lines exhibit a heterogeneous response to Wnt stimulation. (A) Representative immunoblots showing total $\beta$-catenin subcellularlocalization in myeloid cells following CHIR99021 treatment (GSK3 $\beta$ inhibitor). Lamin A/C and $\alpha$-tubulin indicate the purity/loading of the nuclear (N) and cytosol (C) fractions, respectively. (B) Representative flow cytometric histograms showing intensity of the TCF-dependent expression of YFP from the ' $\beta$-catenin activated reporter' (BAR) reporter, or negative control 'found unresponsive $\beta$-catenin activated reporter' (fuBAR) control (containing mutated promoter binding sites) following treatment with CHIR99021/vehicle control [dimethyl sulfoxide (DMSO)] as above. (C) Summary showing the relative percentage nuclear $\beta$-catenin localization (as a proportion of the total) induced in myeloid cell lines upon CHIR99021 treatment. (D) Summary showing the median fluorescence intensity generated from the BAR/fuBAR reporters in myeloid cell lines treated \pm CHIR99021. $* P<0.05 ; * * * * P<0.0001$; ns: not significant. MFI: mean fluorescence intensity. 
binding site showed no induction (Figure 1B). In contrast, ML-1 and U937 cells had a highly restricted capacity for nuclear $\beta$-catenin localization (vs. K562 and HEL cells) despite substantial cytosolic stabilization of the protein (Figure 1B and C). Consistent with this, CHIR99021 treatment of ML-1 or U937 cells caused no detectable activation of the TCF reporter (Figure 1B and 1D). The same patterns of Wnt-responsiveness were observed when cell lines were treated with rWnt3a (Online Supplementary Figure S2). These findings demonstrate that the ability of some leukemia cells to drive a transcriptional response to Wnt agonist is limited by their capacity to permit the nuclear accumulation of $\beta$-catenin; we termed these cells Wnt-unresponsive.

\section{$\beta$-catenin interactome analyses reveal contrasting protein interactions between Wnt-responsive and Wnt-unresponsive leukemia cell lines}

Given the wealth of factors previously reported to regulate the nuclear localization of $\beta$-catenin, ${ }^{3}$ we designed an interactome screen of cytosolic and nuclear $\beta$-catenin interacting partners from representative Wnt-responsive (K562 and HEL), and Wnt-unresponsive (ML1) cell lines so that we could shortlist candidate factors involved in this process (Figure 2A). Prior to mass spectrometry, we validated the efficiency of $\beta$-catenin co-IP (Figure $2 \mathrm{~B}$ ) from both a positive control for high $\beta$-catenin expression (SW620 colorectal cells containing mutated APC: demonstrating a 4.0- and 4.3-fold enrichment of $\beta$-catenin in cytosol and nuclear fractions, respectively) and in the context of agonist-stabilized $\beta$-catenin (HEL: showing a 6.3and 9.1-fold enrichment). We also confirmed co-IP of a known interactor, TCF-4 (TCF7L2), from the nuclear compartment.

Following MS, raw tandem mass tag (TMT) ratios were processed to generate a set of statistically ranked interactions based on significance of fold-change in protein binding (raw and processed MS data available in Online Supplementary MS data sheets). An extensive profile of $\beta$-catenin interactions were observed in K562 cells (225 significantly enriched cytosolic interactions, 118 significantly enriched nuclear interactions) (Figure $3 \mathrm{~A}$ and $\mathrm{B}$ ). In contrast, a comparatively sparse interaction profile was observed in ML1 cells (38 significantly enriched cytosolic interactions, 26 significantly enriched nuclear interactions) (Figure $3 \mathrm{C}$ and D). An extensive repertoire of $\beta$-catenin interactions was also detected in the other Wnt-responsive cell line analyzed, (HEL; 154 significantly enriched cytosolic interactions, 138 significantly enriched nuclear interactions) (Online Supplementary Figure S3). The sparse nuclear $\beta$-catenin interaction network observed in ML1 cells is perhaps unsurprising given the low levels of nuclear $\beta$-catenin; however, the relative paucity of interactors observed in the cytosol of this line was more surprising given the comparable abundance of $\beta$-catenin in this fraction compared with K562/HEL cells.

Our experimental and bioinformatics strategy was validated by the identification of known $\beta$-catenin interactions (highlighted in green), which were also more abundant in the K562 cells (23 in cytosol, 26 in nucleus) versus the ML-1 cells ( 7 in cytosol, 8 in nucleus). From our significantly enriched interactions (Figure 3 , red dots), we identified several putative novel partners for $\beta$-catenin as summarized for K562 (Figure 4A and B and Online Supplementary Table S2), ML1 (Figure 4C and D and Online
Supplementary Table S2), and for HEL (Online Supplementary Table S3 and Online Supplementary Figure S4). Within these significant interactions we identified a number of novel associations of particular interest to myeloid leukemias and/or Wnt signaling which appeared in one or more cell lines (red asterisks), but were outside the remit of this study to investigate further. MBD3 and PRC1 have been found to co-operate with the oncogenic fusion proteins $\mathrm{PML} / \mathrm{RAR} \alpha^{14}$ or PLZF/RAR $\alpha^{15}$ in acute promyelocytic leukemia (APL), and regulate stemness through Wnt/ $\beta$ catenin signaling. ${ }^{16,17}$ The RNA binding protein MSI2 predicts poor prognosis in $\mathrm{AML},{ }^{18}$ more aggressive CML, ${ }^{19}$ and can promote cancer via Wnt signaling. ${ }^{20}$ LIN28B, a microRNA-binding protein, is over-expressed in multiple leukemias including $\mathrm{AML},{ }^{21}$ where it promotes proliferation, ${ }^{22}$ and co-operates with Wnt signaling to drive malignancy. ${ }^{23}$ DDX10, RBM6 and RBM15 are known to form oncogenic fusion proteins in myeloid leukemias, ${ }^{24-26}$ and DDX10 and RBM15 also have roles in promoting Wnt signaling. ${ }^{27,28}$ PUM2 and MKRN2 are two further proteins reported to promote the growth of both normal and malignant hematopoietic cells. ${ }^{29,30} \mathrm{We}$ also confirmed the first reported $\beta$-catenin interaction with Wilms Tumor-1 (WT1) by MS and immunoblotting (Online Supplementary Figure S5), which is of considerable interest in leukemia biology given its frequent dysregulation in AML and association with adverse patient survival. ${ }^{31}$

\section{Nuclear LEF-1 expression correlates with nuclear $\beta$-catenin localization in cell lines and primary acute myeloid leukemia patient cells}

We next examined the data from the fractions of Wntresponsive cells for candidate proteins that could promote the nuclear-localization of $\beta$-catenin. Several had previously been implicated in negative regulation of $\beta$-catenin nuclear localization, GSK $3 \beta,{ }^{32} \alpha$-catenin, ${ }^{33}$ Axin $1 / 2,{ }^{34}$ and APC, ${ }^{34}$ whilst TCF- $4^{34}$ and LEF- ${ }^{35}$ are nuclear localized transcription factors that bind $\beta$-catenin. To validate the MS data, we examined the protein expression of these candidates in a panel of myeloid cell lines by immunoblotting. With the exception of APC, which is problematic to blot, ${ }^{36}$ immunoblotting confirmed $\mathrm{MS}$ analysis in that expression of the negative regulators was mostly limited to Wnt-responsive cells making it unlikely they were responsible for restricted nuclear $\beta$-catenin in Wnt-unresponsive cells (Figure 5A).

We next examined the expression of two known nuclear $\beta$-catenin interactors, TCF-4 and LEF-1, which are ubiquitously expressed in multiple tissues. Both of these proteins are predominantly nuclear in Wnt-responsive cell lines and were absent from the Wnt-unresponsive lines (Figure 5A; matching the proteomics data in Figure 3 and Online Supplementary Figure S3). Of these two proteins, LEF-1 bound $\beta$-catenin with higher significance than TCF4 in both K562 and HEL nuclei. This, together with our previous observation that overexpression of TCF-4 actually suppressed $\beta$-catenin-dependent transcription ${ }^{37}$ (and thus is unlikely to promote nuclear $\beta$-catenin level), led us to focus our investigation on LEF-1. This protein is known to be dysregulated in $\mathrm{AML}^{38}$ so we examined the clinical relevance of this by correlating relative LEF-1 nuclear localization with that of $\beta$-catenin in primary AML blasts. In our cohort of 23 nuclear/cytosol fractionated AML patient samples, we observed a highly significant degree of correlation (Spearman Rank $\mathrm{R}=0.63, P<0.005$ ) between the rel- 
ative nuclear-localized (as a proportion of total) $\beta$-catenin protein and relative nuclear-localized (as a proportion of total) LEF-1 protein (Figure 5B and C). Interestingly, the overall frequency of $\beta$-catenin protein overexpression (approx. 80\%) observed in this largely pediatric AML cohort was higher than previous reports (Online Supplementary Figure S6). Taken together, these data indicate that nuclear $\beta$-catenin translocation capacity is linked to LEF-1 expression in myeloid cell lines, and nuclear LEF1 is a strong clinical predictor of nuclear $\beta$-catenin levels in patient-derived AML blasts.
Modulation of LEF-1 expression in myeloid cells regulates nuclear-localization of $\beta$-catenin and alters Wnt-responsiveness

The above data indicated a correlative relationship between nuclear LEF-1 level and capacity for nuclear $\beta$ catenin localization. To demonstrate causation, we examined the effect of modulating LEF-1 expression on $\beta$-catenin localization. Initially, we tested multiple LEF1 shRNA sequences (Online Supplementary Figure S7A) and selected the best (TRCN0000428355) for knockdown of nuclear LEF-1 expression in the Wnt-responsive K562 and
A

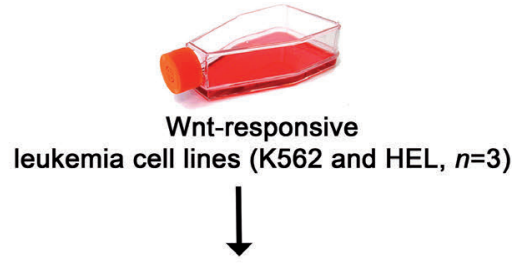

$\beta$-Cat stabilization (CHIR99021 treatment)

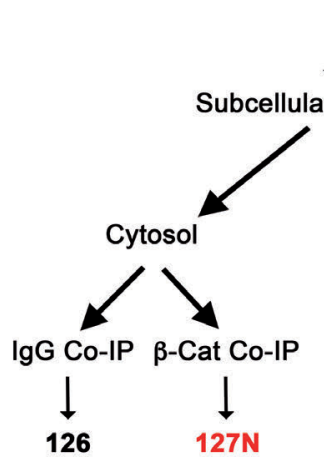

$\downarrow$

ractionation

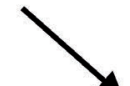

1 Nuclear

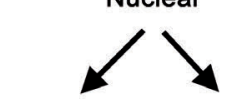

IgG Co-IP $\beta$-Cat Co-IP

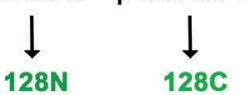

$128 \mathrm{~N} \quad 128 \mathrm{C}$

Tandem Mass Tag (TMT) peptide labeling (8 isobaric mass tags)

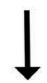

Combine all fractions, fractionate, clean-up, mass spectrometry analysis

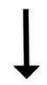

Quantitative identification of $\beta$-catenin cytoplasmic/nuclear protein interactions in myeloid leukemia cells

$\downarrow$

IgG Co-IP $\beta$-Cat Co-IP

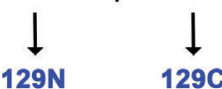

Wnt-unresponsive

leukemia cell line (ML1, $n=3)$

$\beta$-Cat stabilization (CHIR99021 treatment)

Subcellular fractionation
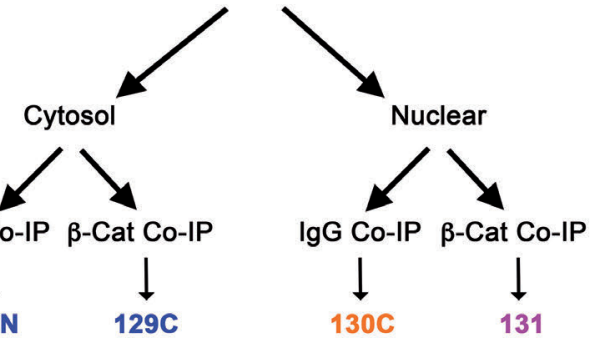

$130 \mathrm{C}$

131

B

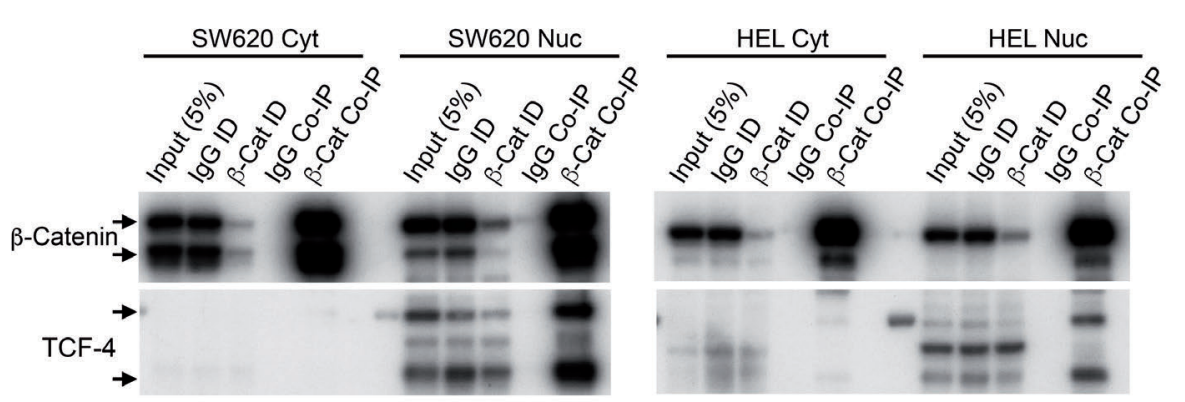

Figure 2. Experimental strategy for analysis of $\beta$-catenin interaction partners in myeloid leukemia cell lines. (A) Wnt-responsive K562/HEL cells and Wnt-unresponsive cells ML-1 cells were treated with CHIR99021 to stabilize $\beta$-catenin prior to cytosolic/nuclear fractionation. From these fractions, either an IgG or $\beta$-catenin ( $\beta$-cat) co-immunoprecipitation (co-IP) was performed generating eight samples which were each TMT labeled with a unique isobaric mass tag. All samples were pooled, fractionated, cleaned and analyzed by mass spectrometry. Mass intensities from each tag report the relative peptide abundance in each sample. Quantitative fold-enrichment of $\beta$-catenin co-IP was obtained by comparison with fraction-matched IgG co-IP control. Three biological replicates were performed on each cell line. (B) Representative immunoblots showing the efficiency of total $\beta$-catenin co-IP performed from the cytosol or nuclear fractions of SW620 cells and HEL cells. Detection of the known interaction partner TCF-4 was used to assess binding partner efficiency. ID: immunodepleted lysate. $\beta$-catenin arrows represent fulllength/degradation intermediates; TCF-4 arrows indicate 58/79kDa transcriptional isoforms. 
HEL cell lines. This approach resulted in a $72 \% \pm 7 \%$ knockdown in LEF-1 protein in the nuclei of K562 cells and an $89 \% \pm 4 \%$ knockdown in HEL cells (Figure 6A). Somewhat lower levels of $L E F-1$ knockdown were observed in CHIR99021-treated cells $(65 \% \pm 19 \%$ and $83 \% \pm 7 \%$, respectively) probably a result of LEF1 being a Wnt target gene and thus being induced through Wnt agonist treatment. ${ }^{39}$ LEF-1 knockdown perturbed nuclear localization of $\beta$-catenin by approximately one-third (28\%) in K562 following CHIR99021 treatment, proportionate to control cells. This reduction was accentuated in HEL cells (41\%) which corresponded to the greater degree of $L E F-1$ knockdown in these cells (Figure 6B). The knockdown of LEF-1 protein resulted in significantly reduced growth of both K562 and HEL cells at multiple time points across a range of serum concentrations (Figure 6C). Use of a second LEF1 shRNA and a different method of Wnt stimulation (rWnt3a) resulted in a similar finding (Online Supplementary Figure $S 7 B$ and $C$ ). These data suggest LEF-1 promotes the optimal translocation of $\beta$-catenin into the nucleus of Wnt-responsive cells and partly contributes to their growth.

Next, we examined whether LEF-1 expression was sufficient to permit nuclear-localization of $\beta$-catenin. To establish this, we stably over-expressed LEF1 in the Wntunresponsive (and LEF-1 negative) U937 and ML1 cells. Overexpression of LEF-1 resulted in substantial cytosolic expression of the full-length LEF-1 protein $(50 \mathrm{kDa})$ but weak nuclear expression; despite this, we observed a dramatic increase in nuclear localized $\beta$-catenin in both ML1 (4-fold) and U937 (2.3-fold) cells over-expressing LEF1 following CHIR99021 treatment (Figure 6D and E). This disparity may be explained by the abundant expression of a short-form of LEF-1 in the nucleus $(25-30 \mathrm{kDa})$ that was absent in Wnt-responsive lines (discussed below). These effects were mirrored using Wnt3a treatment (Online Supplementary Figure S7C) and we also showed that LEF-1 overexpression was able to facilitate nuclear localization of $\beta$-catenin in two further AML cell lines (PLB-985 and THP1) (Online Supplementary Figure S7D). These data demonstrate that overexpression of LEF-1 can significantly increase the capacity for nuclear $\beta$-catenin localization in Wnt-unresponsive cell lines.

To assess the impact of LEF-1 knockdown (and subsequent nuclear $\beta$-catenin reduction) on Wnt signaling, we measured TCF reporter activity. As predicted, Wnt signaling induction in Wnt-responsive K562 and HEL cells following CHIR99021 treatment was severely diminished

\footnotetext{
$\bullet=$ significantly enriched interaction

$-=$ known $\beta$-catenin interaction

$\cdot=\beta$-catenin bait
}

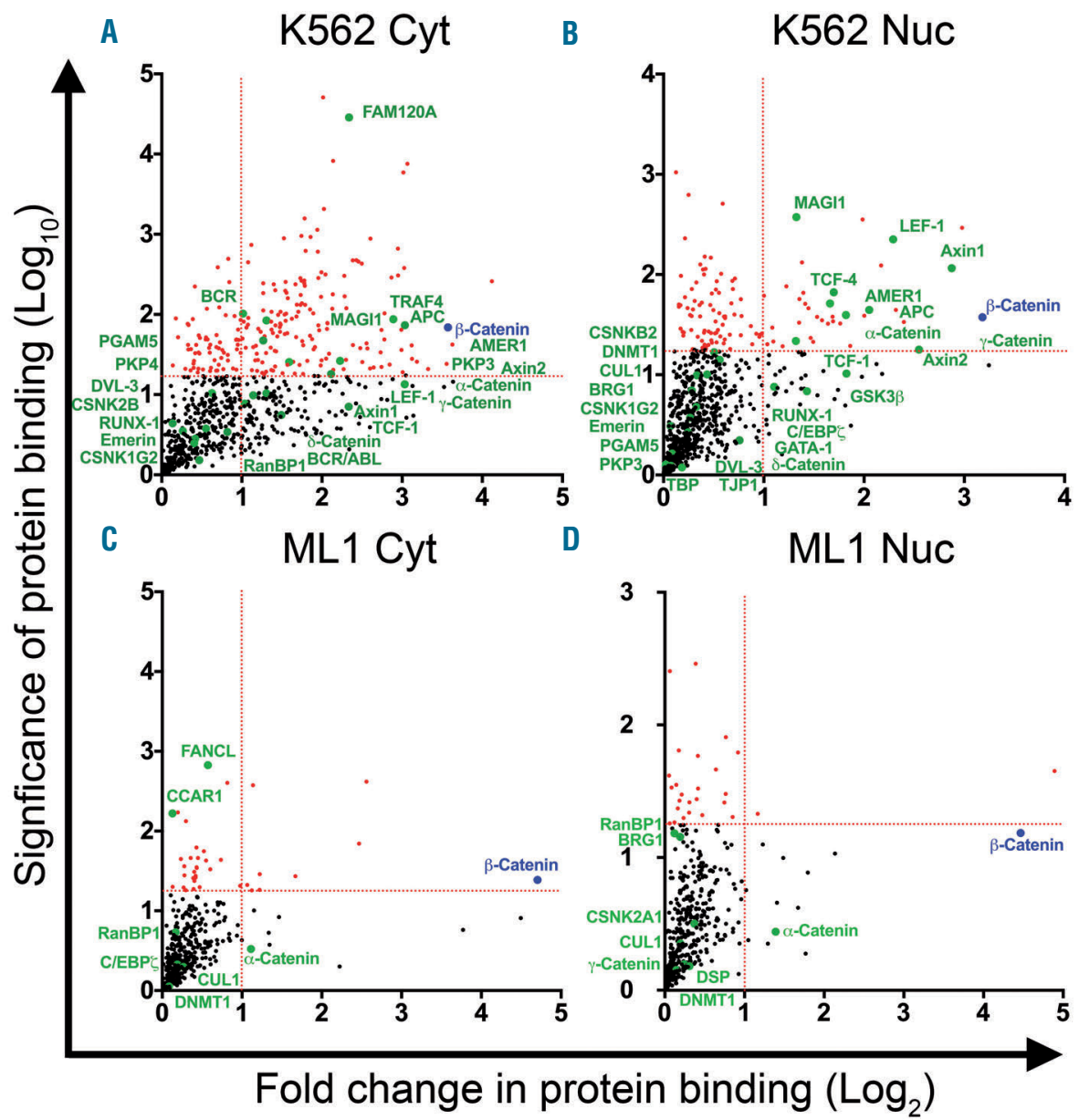

Figure 3. Proteomics analyses reveal contrasting $\beta$-catenin interaction profiles between Wntresponsive and Wnt-unresponsive leukemia cell lines. Scatter plots showing $\beta$-catenin protein interactions detected in (A) K562 cytosolic, (B) K562 nuclear, (C) ML1 cytosolic, and (D) ML1 nuclear fractions. Vertical dashed red line indicates the threshold for 2-fold change in protein binding at $\log _{2}$ (=1) relative to IgG co-immunoprecipitation.. Horizontal red line represents threshold for significant interactions at $P=0.05$ on $\log _{10}$ scale (=1.3). Highlighted red dots indicate statistically significant interactions and green highlighted events/labels indicate known interactions/associations for $\beta$-catenin. Remaining black dots represent other proteins detected in the MS analysis; see also Online Supplementary Data sheets. Fold change values less than 0 are not shown because these likely represent contaminants (see Online Supplementary MS data sheets). 
following $L E F-1$ knockdown (Figure 7A and B). A significant reduction in Wnt signaling output was also observed following use of an alternative LEF1 shRNA in response to CHIR99021 or Wnt3a stimulation (Online Supplementary Figure S8). Assessment of the TCF reporter activity in the LEF1 over-expressing lines was not possible due to the confounding expression of the GFP selectable marker; instead, we examined the protein expression of the classic downstream Wnt target proteins survivin, c-MYC and cyclinD1. We found that LEF1 overexpression significantly enhanced the expression of these proteins following CHIR99021 treatment (Figure 7C and D), indicating that

A

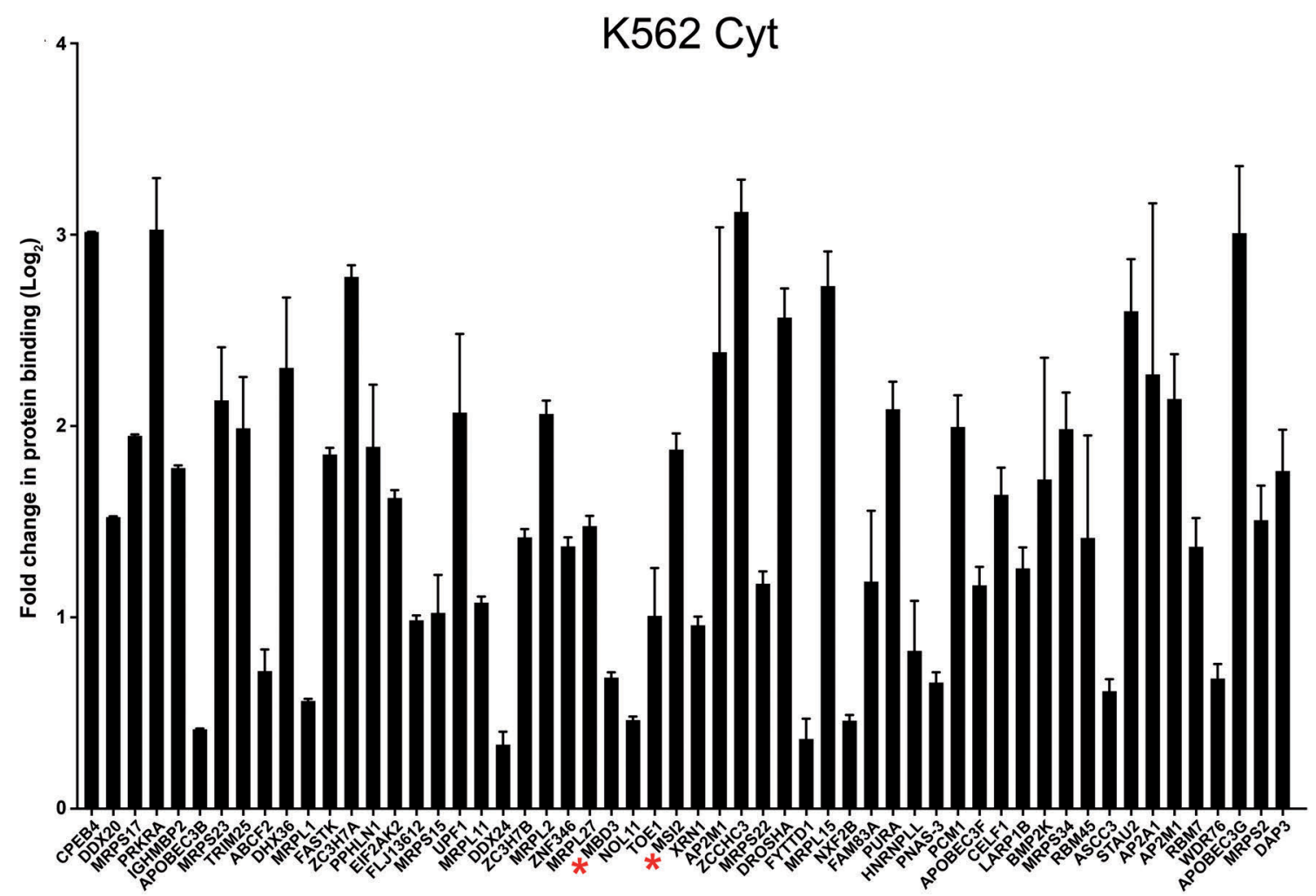

B

K562 Nuc

C

ML1 Cyt

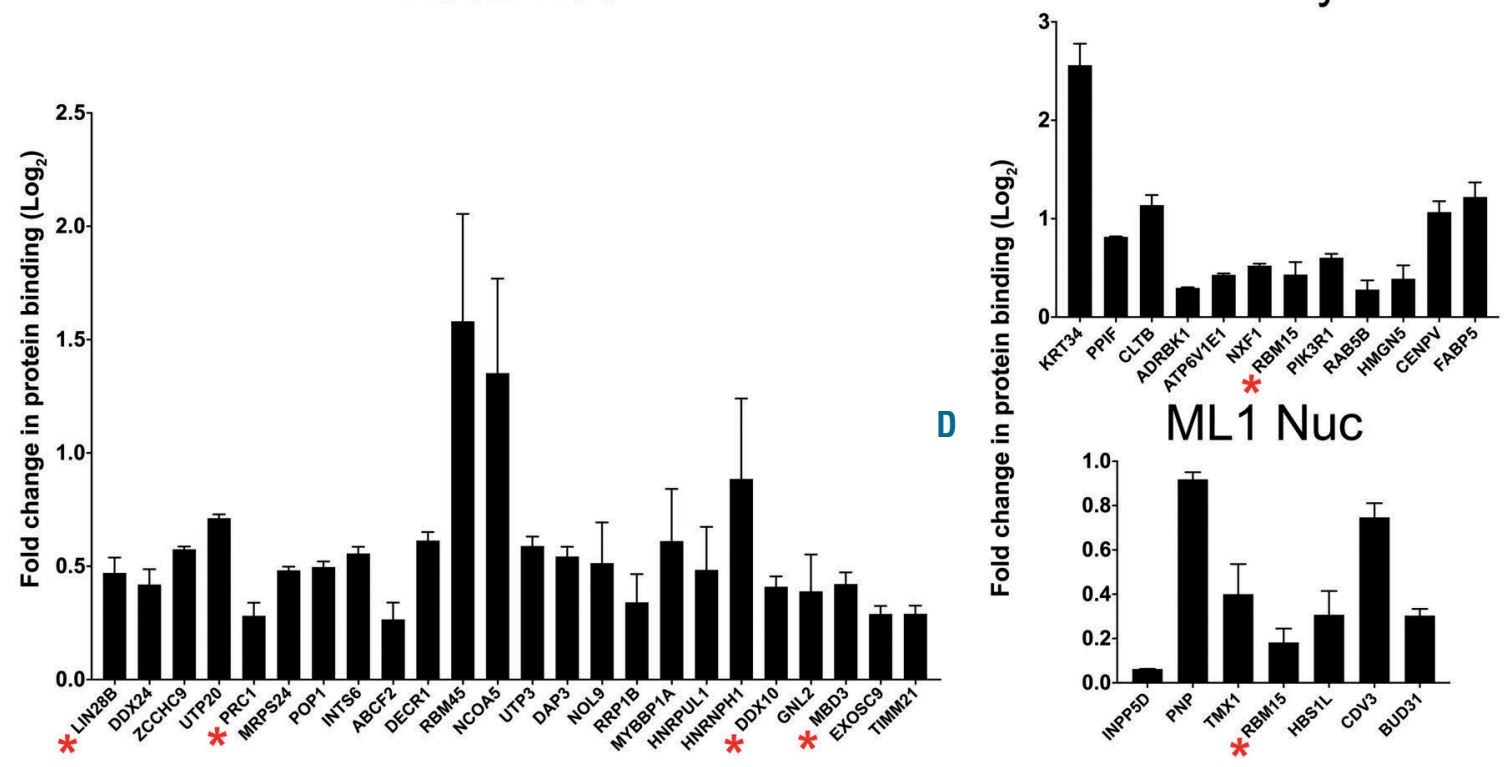

Figure 4. Mass spectrometric analyses identify putative novel interaction partners for $\beta$-catenin in myeloid leukemia cell lines. Bar graphs summarizing the average fold change in protein binding (relative to matched IgG co-immunoprecipitation) for novel, significant $\beta$-catenin interactions observed in (A) K562 cytosolic, (B) K562 nuclear, (C) ML1 cytosolic, and (D) ML1 nuclear fractions. Significant proteins only are shown (red dots in Figure 3 panels) with a frequency on the CRAPome database of $\leq 10 \%$ and known interactions were removed. Red asterisks represent proteins of particular significance to myeloid leukemias and/or Wnt signaling (see Results). Proteins are ranked along x-axis according to statistical significance (most significant on the left) (see also Online Supplementary Table S2). 
the observed translocation of $\beta$-catenin was sufficient to generate a transcriptional response. A caveat to these data is that these observations cannot be linked exclusively to the modulation of $\beta$-catenin nuclear-localization since LEF-1 expression is a co-variable in these experiments. Taken together, these data indicate LEF-1 can regulate the nuclear level of $\beta$-catenin and our observations are also consistent with a concomitant regulation of Wnt signaling activity.

\section{LEF-1 protein is proteolytically cleaved in Wnt-unre- sponsive cells}

In the experiments above, overexpression of full-length LEF-1 in Wnt-unresponsive cell lines resulted in the emergence of a $25-30 \mathrm{kDa}$ species of LEF-1 protein. To evaluate the contribution of this short LEF-1 form with the increased nuclear $\beta$-catenin level observed in these cell lines we examined the $\beta$-catenin binding capacity of this species. We performed $\beta$-catenin co-IP from both the cytosol and nucleus of CHIR99021 treated LEF1-overexpressing U937 cells and immunoblotted for LEF-1 protein. Both the full-length and short-forms of LEF-1 protein co-immunoprecipitated with $\beta$-catenin from the cytosol implying both forms bind $\beta$-catenin in this cell line. In the nuclear fraction, $\beta$-catenin preferentially co-immunoprecipitated with the short LEF-1 form, though the proportion of short-form was highly enriched in this fraction (Figure 8A; input lane). These data confirm that the short- form LEF-1 has $\beta$-catenin binding ability and could mediate the increased nuclear $\beta$-catenin translocation observed in Wnt-unresponsive cell lines above upon LEF-1 overexpression with CHIR99021 treatment.

Finally, we examined the origin of the short LEF-1 polypeptide. This variant was unlikely to have derived from alternative splicing since the ectopic expression of LEF-1 was driven from cDNA. Furthermore, these short forms of LEF-1 were also observed endogenously in colorectal cell lines (Online Supplementary Figure S9A) and in primary AML samples featured in Figure 5 (full blots in Online Supplementary Figure S9B). Therefore, the presence of short-form LEF-1 was most consistent with a proteolytic cleavage mechanism. To investigate this, nuclear lysates from both K562 control and U937-LEF1 cells \pm protease inhibitor cocktail (PIC) were incubated at $37^{\circ} \mathrm{C}$ and the relative proportions of full-length to short LEF-1 forms in each cell line observed over 0-60 min. In K562 control cells, full-length LEF-1 protein was stable with no detectable breakdown of full-length LEF-1 protein even in the absence of PIC (Figure 8B). In contrast, the full-length LEF-1 band present in U937-LEF-1 cells was reduced with concomitant enrichment of the short-form polypeptide after a $10 \mathrm{~min}$ incubation at $37^{\circ} \mathrm{C}$ (Figure $8 \mathrm{C}$ ). The removal of the PIC reduced the half-life of full-length LEF-1 by approximately $50 \%$ with degradation occurring within 5 min. Together these data are consistent with the shortform of LEF-1 arising through proteolytic processing.
A

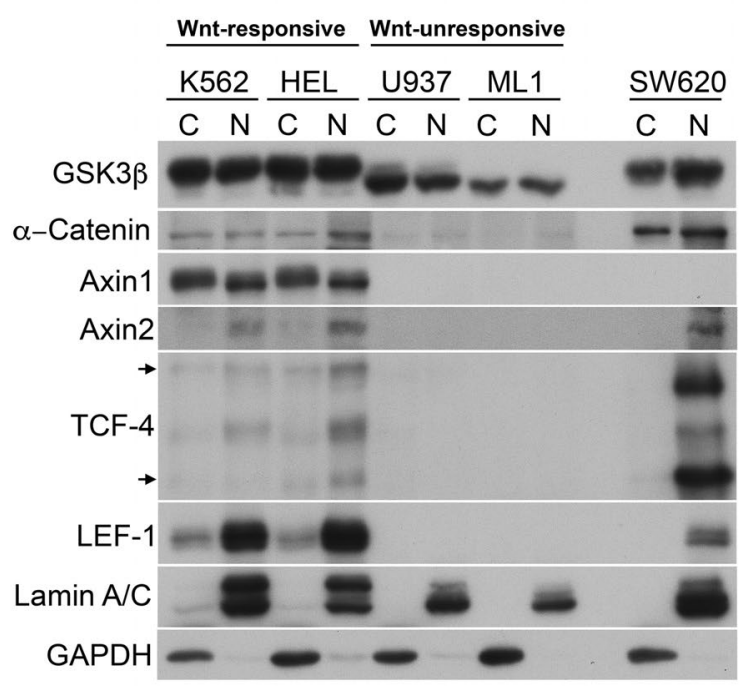

B
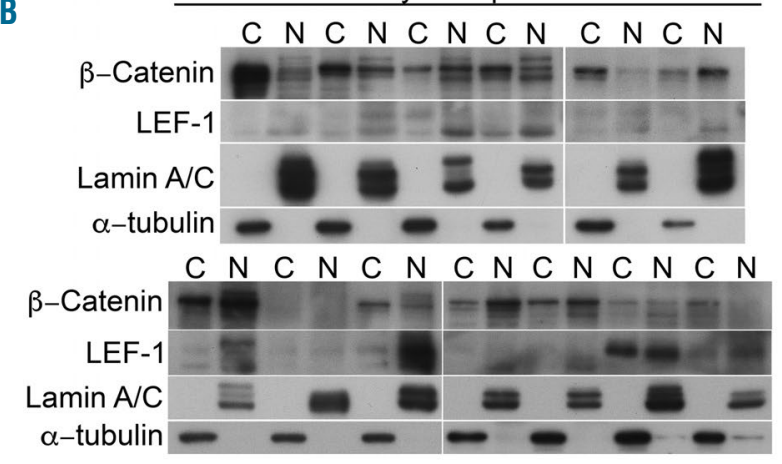

c

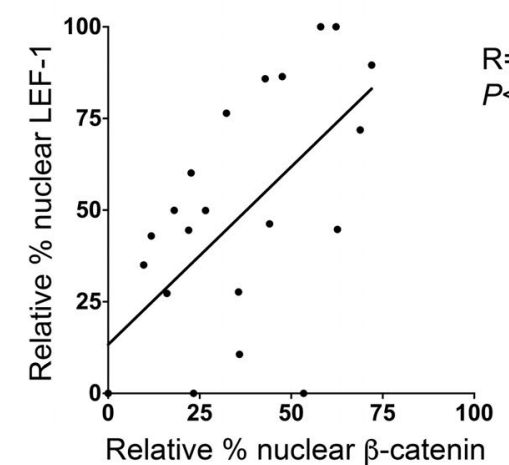

Figure 5. Correlation between nuclear LEF-1 and nuclear $\beta$-catenin in leukemia cell lines and primary acute myeloid leukemia (AML) samples. (A) Representative immunoblot screen of myeloid leukemia cell lines showing the relative cytoplasmic $(C)$ and nuclear $(\mathrm{N})$ abundance of candidate proteins influencing $\beta$-catenin nuclear localization detected by mass spectrometry. SW620 colorectal cells were used as a positive control. TCF-4 arrows indicate 58/79kDa transcriptional isoforms. (B) Representative immunoblots showing the cytosolic and nuclear expression of both LEF-1 and total $\beta$-catenin in primary AML patient samples. LEF-1 protein isoforms migrate as bands between 40-55kDa. Lamin A/C and GAPDH or $\alpha$-tubulin were used to assess fractionation efficiency and equal protein loading. (C) Summary scatter plot showing correlation between relative percentage nuclear $\beta$-catenin localization and relative percentage nuclear LEF-1 localization in primary AML patient blasts. 
A

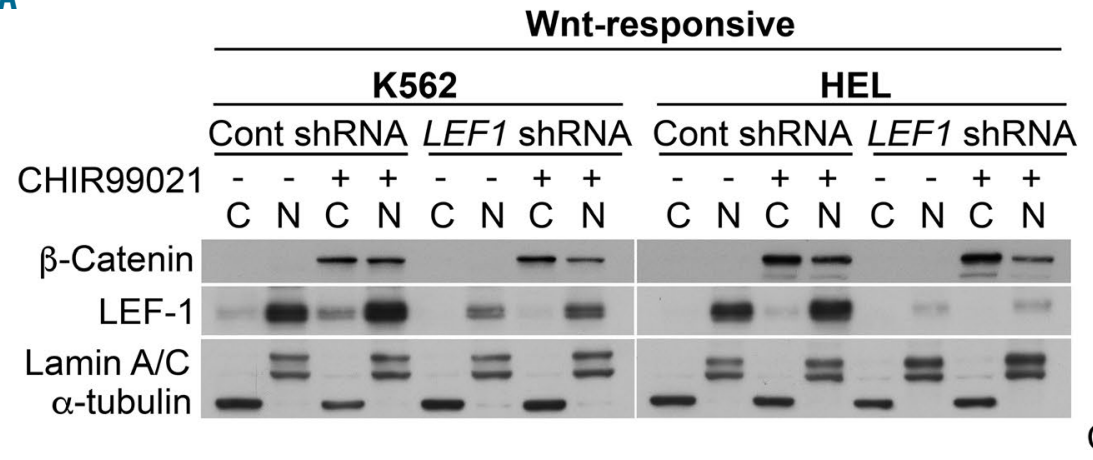

C

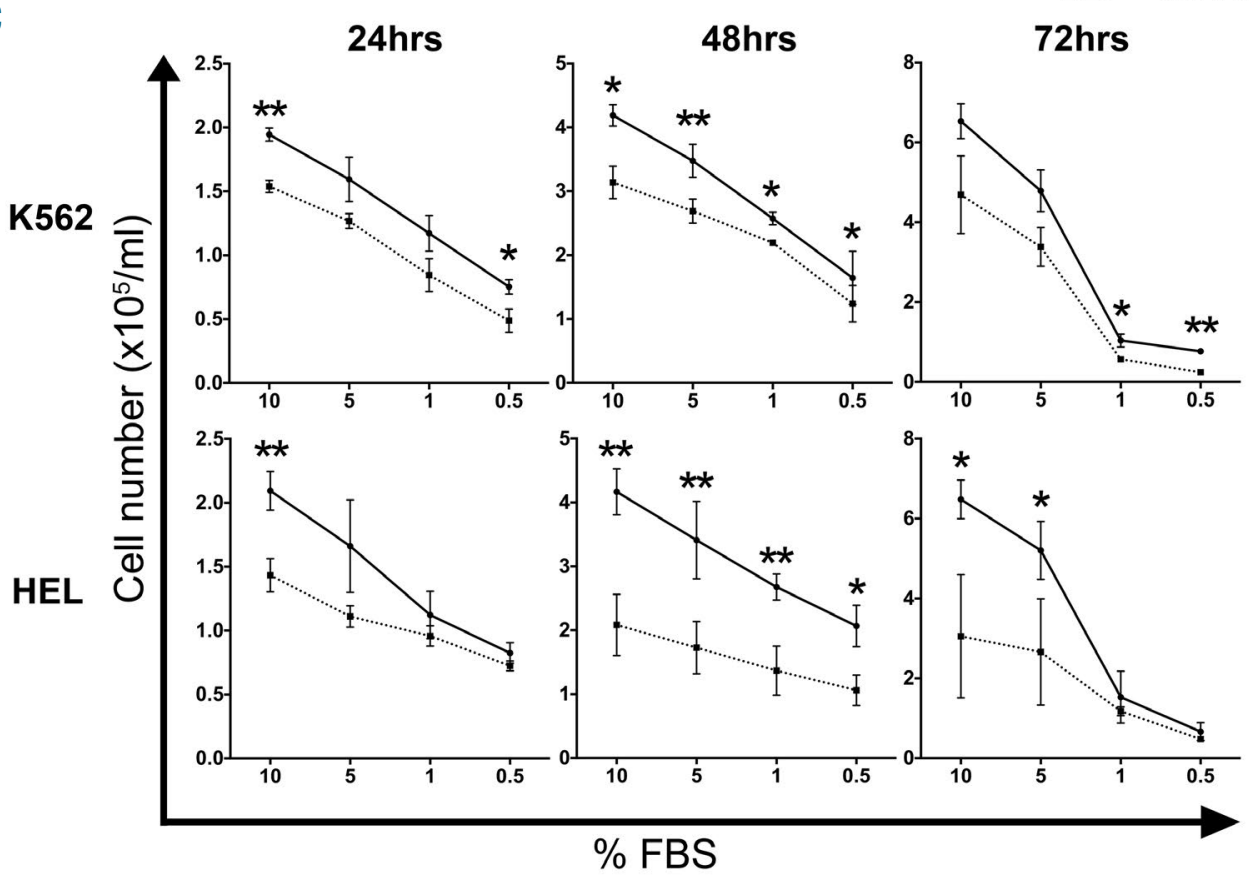

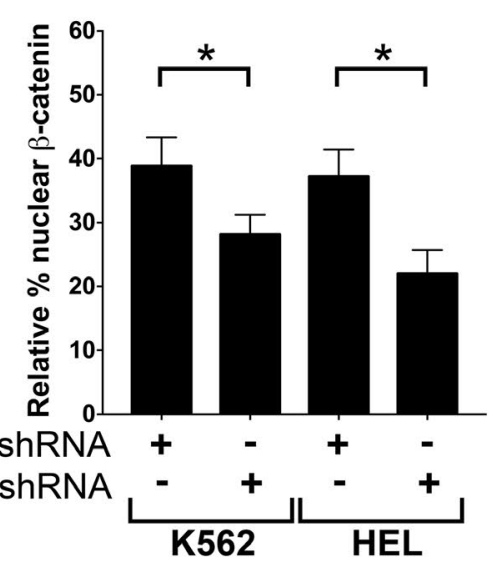

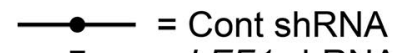

D

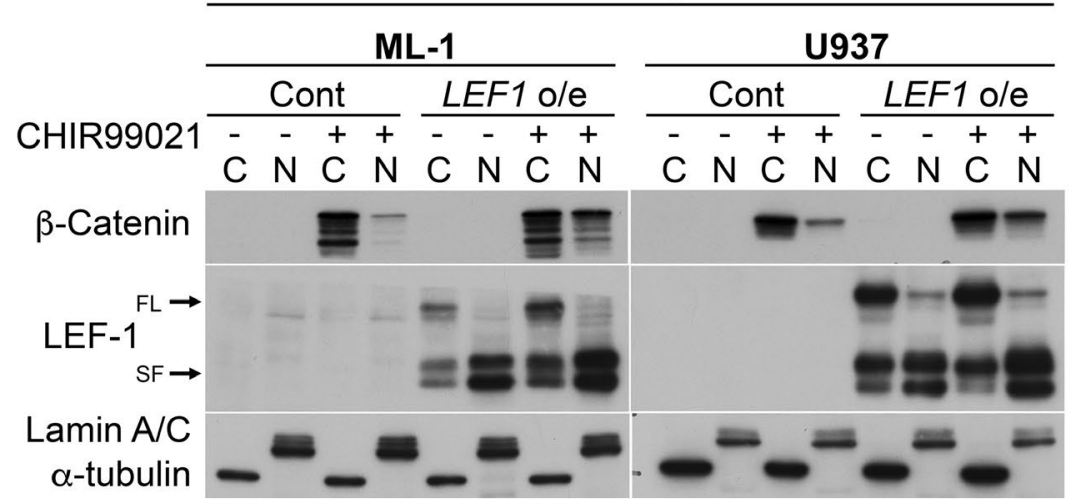

$\mathrm{E}$

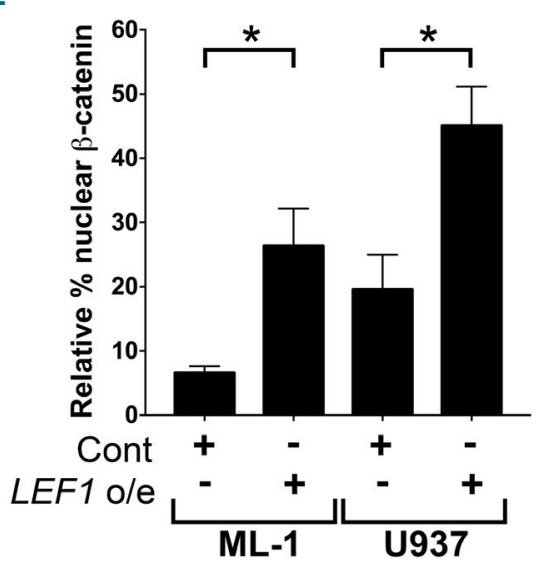

Figure 6. Modulation of LEF-1 expression affects relative nuclear $\beta$-catenin level. (A) Representative immunoblots showing the level and subcellular localization of total $\beta$-catenin and LEF-1 protein in K562 and HEL cells in response to control/LEF1 shRNA \pm CHIR99021 treatment. Shorter 6-hour (hrs) CHIR99021 treatments were used for these cells to minimize the LEF-1 expression induced through Wnt stimulation. (B) Summary graph showing the relative percentage nuclear $\beta$-catenin localization induced upon 6-hr CHIR99021 treatment of K562 or HEL cells \pm control/LEF-1 shRNA. (C) Effect of LEF1 shRNA on the proliferation of K562 and HEL at different concentrations of serum after 24,48 and 72 hrs. (D) Representative immunoblots showing the level and subcellular localization of total $\beta$-catenin and LEF1 protein in ML-1 and U937 cells in response to control/LEF1 overexpression (o/e) \pm 16 -hrs CHIR99021 treatment. The positions of full-length (FL) and short-forms (SF) of LEF-1 protein on the blot are indicated by arrows. Lamin A/C and $\alpha$-tubulin were used to assess fraction purity and protein loading. (E) Summary graph showing the relative percentage nuclear $\beta$-catenin localization induced upon CHIR99021 treatment of ML-1 and U937 cells for 16 hrs \pm control/LEF1 overexpression. $\star P<0.05 ; * * P<0.01$. 


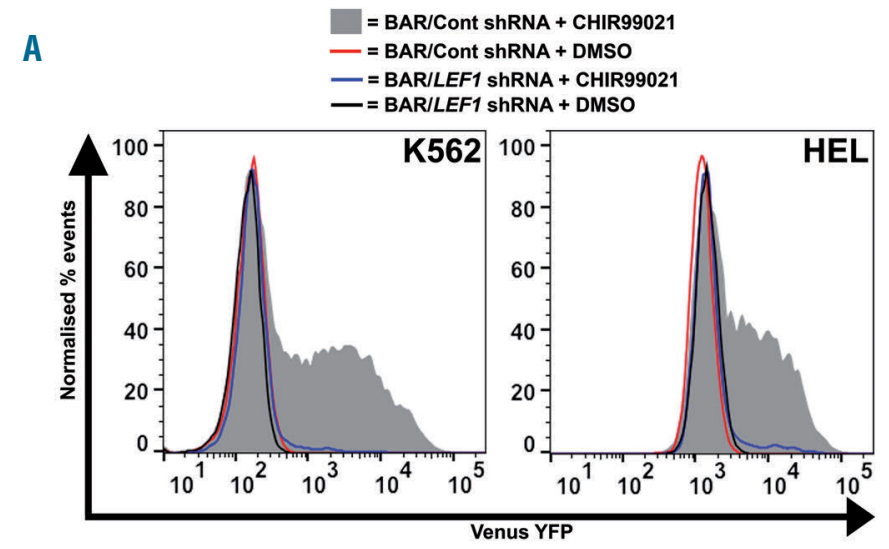

C

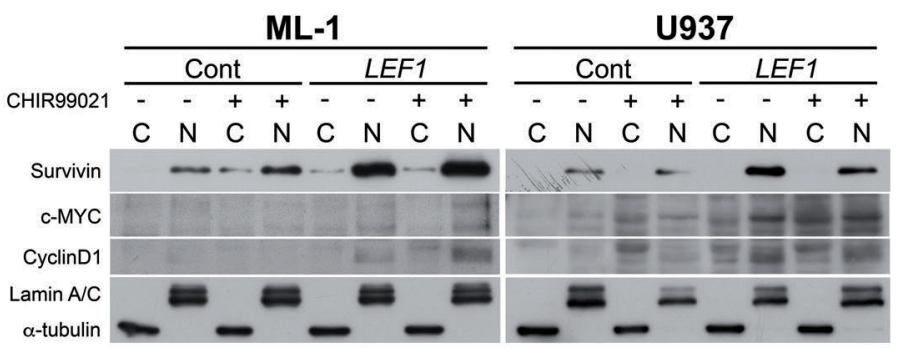

B

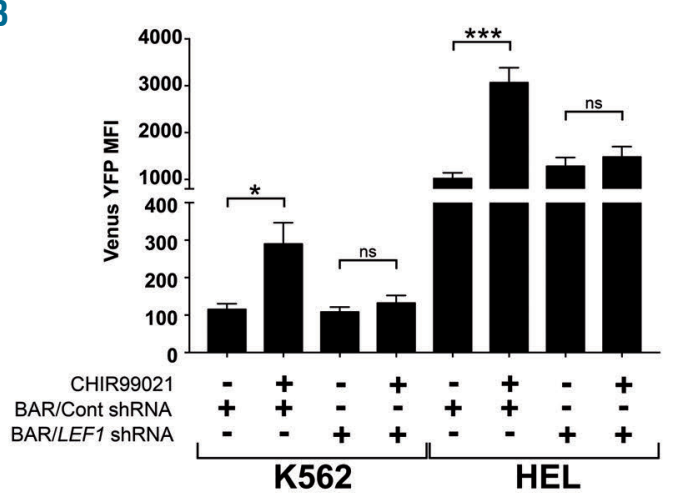

D

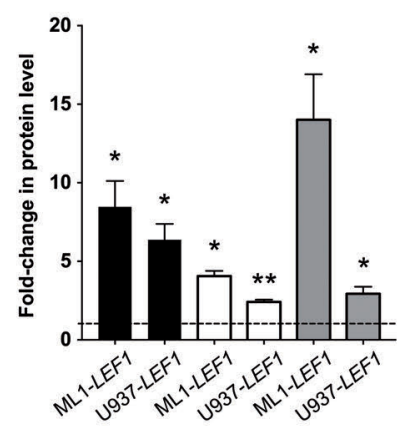

Figure 7. Modulation of LEF-1 expression affects downstream Wnt signaling. (A) Representative flow cytometric histograms showing intensity of the TCF reporter (BAR) in K562 and HEL cells treated with control/LEF1 shRNA \pm CHIR99021. (B) Summary data showing the median fluorescence intensity generated from the BAR reporter in K562 and HEL cells treated with control/LEF1 shRNA \pm CHIR99021. (C) Representative immunoblots showing expression of known Wnt target proteins survivin, c-MYC and cyclinD1 in ML-1 and U937 cells in response to control/LEF1 overexpression \pm CHIR99021. Lamin A/C and $\alpha$-tubulin were used to assess fraction purity and protein loading. (D) Summary data showing the relative fold-change in nuclear protein expression of classic Wnt targets survivin, C-MYC and cyclinD1 in CHIR99021 treated ML1 and U937 cells over-expressing LEF1. Dashed line represents relative level (=1) present in nuclei of CHIR99021-treated ML1 and U937 cells expressing control plasmid. $* P<0.05 ; * * P<0.01 ; * * * P<0.001$. ns: not significant.

A U937-LEF1 Cyt U937-LEF1 NuC

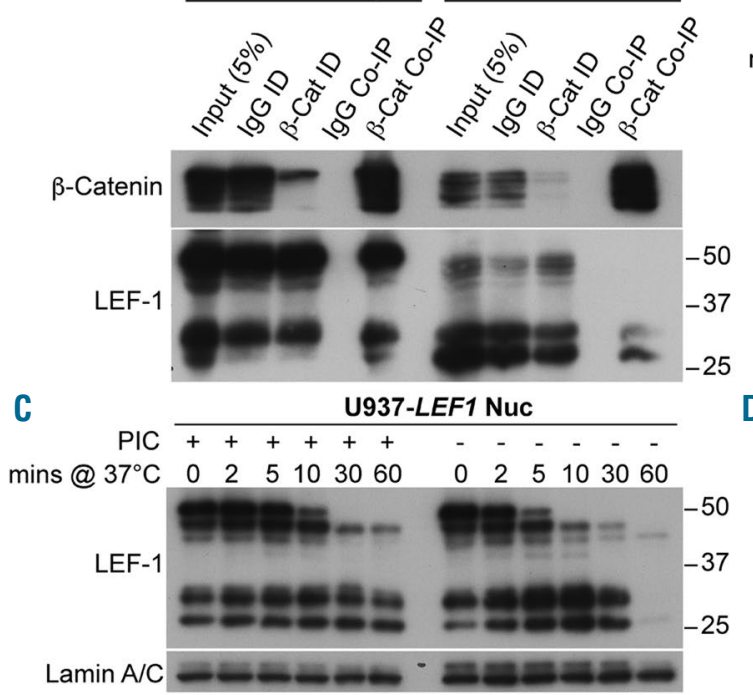

\section{B} K562 Control Nuc

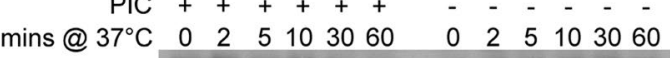

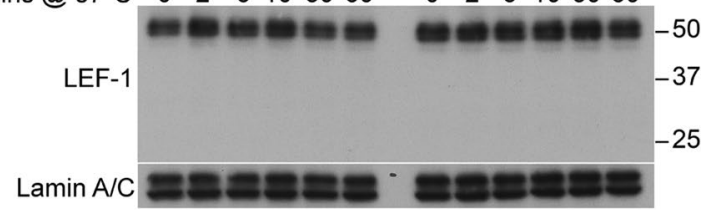

K562 Cont Nuc + U937 Cont whole (1:1) D

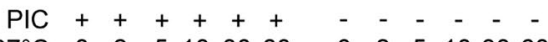
mins@ @ $37^{\circ} \mathrm{C} \quad 0 \begin{array}{llllllllllll}0 & 0 & 10 & 30 & 60 & 0 & 2 & 5 & 10 & 30 & 60\end{array}$

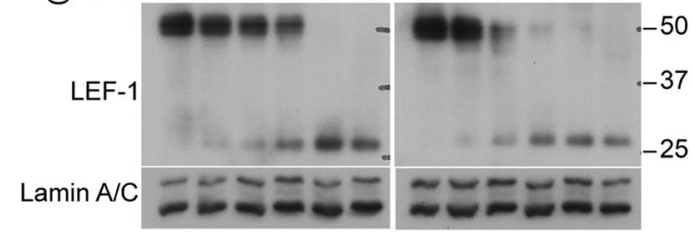

Figure 8. Short-form LEF-1 is proteolytically-derived and is capable of $\beta$-catenin binding. (A) Representative immunoblot showing total $\beta$-catenin co-immunoprecipitation (Co-IP) from cytosolic and nuclear fraction of LEF1-over-expressing U937 cells. Total $\beta$-catenin and LEF-1 protein levels are shown. ID: immunodepleted lysate. Representative immunoblot showing LEF-1 protein levels in (B) K562 control and (C) U937-LEF1 nuclear lysates \pm protease inhibitor cocktail (PIC) during time course incubation at $37^{\circ} \mathrm{C}$. (D) Representative immunoblots showing LEF-1 protein levels in K562 control nuclear lysate after mixing with U937 control whole cell lysate (1:1 protein concentration) $\pm \mathrm{PIC}$ during time course incubation at $37^{\circ} \mathrm{C}$. Nuclear lamin $\mathrm{A} / \mathrm{C}$ detection was used to assess protein loading. 
These data suggest that LEF-1-targeted proteases are active in Wnt-unresponsive U937 cells, but inactive/absent in Wnt-responsive K562 cells. To test this, we mixed nuclear lysates from K562 cells directly with whole cell lysates from U937 control cells (which are LEF1 negative). Remarkably, the previously stable full-length LEF-1 band present in K562 cells exhibited marked and rapid reduction with a concomitant increase in the shortform polypeptide, a process exacerbated by PIC removal (Figure 8D). These data confirm that constitutive LEF-1 degradation mechanisms are active in Wnt-unresponsive cells but are absent/inhibited in Wnt-responsive cells.

\section{Discussion}

Canonical Wnt signaling has emerged as one of the most frequently dysregulated signaling pathways in myeloid neoplasms which has led to considerable interest in targeting this pathway. The central mediator $\beta$-catenin represents an appealing therapeutic target because of its leukemogenic role, ${ }^{6,7}$ prognostic influence, ${ }^{5}$ and functional redundancy in normal hematopoietic development. ${ }^{40}$ The characterization of the hematopoietic interactome of $\beta$ catenin is, therefore, of considerable interest, and the experimental approach adopted in this study was validated by the identification of multiple known $\beta$-catenin partners, such as Axin, TCF-4, $\alpha$-catenin and APC. Associations with key hematopoietic transcription factors, C/EBP' and GATA-1, were identified and these are known to co-occupy genomic sites with TCF-4 (a known $\beta$-catenin partner) during hematopoietic development. ${ }^{41}$ We confirmed the previously reported interaction of $\beta$ catenin with the oncogenic fusion protein BCR-ABL in CML, which is present in K562 cells. ${ }^{9}$ In addition to known partners, we identified putative novel interactions which have known relevance to leukemia and/or Wnt signaling (MBD3, ${ }^{14,16}$ PRC1, ${ }^{15,17}$ MSI2, ${ }^{18-20}$ LIN28B, ${ }^{21-23}$ DDX10, ${ }^{24,27} \mathrm{RBM6}^{25} \mathrm{RBM}^{15,26,28}$ PUM2, ${ }^{29}$ MKRN2 ${ }^{30}$ and WT1 $\left.{ }^{31}\right)$.

To investigate the nuclear localization mechanisms of $\beta$-catenin, we focused on LEF-1, given that it was one of the most significantly enriched interactors present in Wnt-responsive cell nuclei, and its nuclear expression was highly predictive of $\beta$-catenin nuclear-localization in both myeloid cell lines and primary AML blasts. A role for LEF1 in mediating nuclear-localization of $\beta$-catenin was confirmed using both knockdown and overexpression approaches. LEF-1 contains a nuclear localization sequence and is known to shuttle between cytoplasmic and nuclear compartments. ${ }^{42}$ This suggests LEF-1 could serve as a cytosolic-nuclear chaperone for $\beta$-catenin. However, the high nuclear:cytosol ratio of LEF-1 expression observed in Wnt-responsive cells (K562 and HEL) would be more consistent with LEF-1 serving as a nuclear retention factor for $\beta$-catenin, as has previously been demonstrated for other Wnt signaling components. ${ }^{34}$ This is the first evidence of LEF-1 contributing to $\beta$-catenin nuclear-localization capacity in human myeloid leukemia cells; however, our data cannot exclude the role of other factors in this process. Nuclear export mechanisms may also be influential in limiting nuclear accumulation of $\beta$-catenin in Wnt-unresponsive cell lines. In particular, RanBP1 was an interacting partner detected for $\beta$-catenin in ML-1 nuclei but absent in K562/HEL nuclei, and may warrant further study given the documented role for RanBP3 in mediating $\beta$-catenin export. ${ }^{43}$ Two factors recently implicated in nuclear $\beta$-catenin regulation including RAPGEF5 ${ }^{44}$ and Twa $1^{45}$ were not detected in our analyses, suggesting their interaction with $\beta$-catenin is context-dependent.

A strong correlation between the relative levels of nuclear localized $\beta$-catenin and LEF-1 was identified in primary AML samples suggesting this relationship may have clinical relevance. Indeed, expression of constitutively active LEF-1 in HSPC induced AML in mice, ${ }^{38}$ and LEF-1 expression promotes the survival of myeloid leukemia cell lines. ${ }^{46}$ In our study, we observed significantly inhibited growth in K562 and HEL cells harboring LEF-1 knockdown (Figure 6C). Given the adverse prognosis of nuclear $\beta$-catenin in $\mathrm{AML}^{5}$ and recent studies demonstrating its therapeutic merit in AML models, ${ }^{47-49}$ LEF-1 represents an attractive target in myeloid leukemia since it also inhibits $\beta$-catenin nuclear localization. Small molecule inhibitors of $\beta$-catenin: TCF/LEF interaction are under development and have shown efficacy in leukemia treatment. ${ }^{50-52}$ It would be interesting to observe if this efficacy is partly driven by reducing the level of nuclear $\beta$ catenin in leukemia cells. This study focused on the role of LEF- 1 driving aberrant $\beta$-catenin nuclear-localization in myeloid leukemia; however, given the frequency of Wnt/ $\beta$-catenin dysregulation in human cancer, this axis could be active in other malignancies.

We also observed that LEF-1 is proteolytically degraded in Wnt-unresponsive leukemia cells resulting in the emergence of short-form LEF-1 proteins $(25-30 \mathrm{kDa})$. These were smaller than the $38 \mathrm{kDa}$ transcriptional isoforms previously reported to derive from alternative splicing or alternative promoter usage $\mathrm{e}^{39,53}$ which serve as dominantnegative inhibitors of Wnt signaling because they lack the $\beta$-catenin binding domain necessary to initiate transcription. ${ }^{39}$ In contrast, the proteolytic fragments observed in this study retained $\beta$-catenin binding capacity (Figure 7A) and did not appear to have dominant-negative function (Wnt targets were still activated) (Figure 6C and D). Under the conditions of the experiment, however, where LEF-1 is being over-expressed, the abundance of these fragments may have a dominant effect on $\beta$-catenin retention in the nucleus, whereas under normal circumstances, it is likely that degradation serves to remove LEF1 and suppress the nuclear retention of $\beta$-catenin. This post-translational regulation of LEF-1 has parallels with embryonic stem cells where proteolytic cleavage of TCF3 (TCF7L1), a closely related family member, removes it from target genes when differentiation signals trigger the suppression of Wnt signaling. ${ }^{54}$ In leukemia cells, this proteolytic cleavage could be mediated by NLK (Nemo-like kinase) which binds the E3 ubiquitin-ligase NARF (NLK associated RING finger protein) and reportedly induces ubiquitylation (and proteasomal degradation) of LEF-1 in co-operation with the E2 conjugating enzyme E2-25K, ${ }^{55}$ a mechanism previously reported to be active in leukemia cells. ${ }^{56}$

In summary, our study has made three key findings: firstly, the generation of the first $\beta$-catenin interactomes in leukemia cells; secondly, the characterization of LEF-1 as a regulator of nuclear $\beta$-catenin localization in leukemia; and, finally, the demonstration of post-transcriptional proteolytic degradation mechanisms for controlling LEF-1 expression in myeloid leukemia cells. 


\section{Acknowledgments}

RGM is funded by a Kay Kendall Leukemia Fund (KKLF) Junior Fellowship (KKL1051), British Society for Haematology (BSH) Early Stage Research Start-up Grant (34725), and a Royal Society Research Grant (RG160682). JR is funded by Cancer Research Wales. AT and RLD received funding from a Bloodwise programme grant (13029). We are grateful to clini- cal staff, including Lizzy Palmer and Julia Wolf, at the Bristol Royal Hospital for Children (BRHC) and the Bristol Haematology and Oncology Centre (BHOC) respectively, for collection of $A M L$ patient samples and supplying associated clinical data. We are also grateful to the patients and their families who gave permission for their cells to be used for research.

\section{References}

1. Nusse R, Clevers H. Wnt/beta-Catenin Signaling, Disease, and Emerging Therapeutic Modalities. Cell. 2017;169(6): 985-999.

2. Li VS, Ng SS, Boersema PJ, et al. Wnt signaling through inhibition of beta-catenin degradation in an intact Axin1 complex. Cell. 2012;149(6):1245-1256.

3. Morgan RG, Ridsdale J, Tonks A, Darley RL. Factors affecting the nuclear localization of beta-catenin in normal and malignant tissue. J Cell Biochem. 2014;115(8):1351-1361.

4. Simon M, Grandage VL, Linch DC, Khwaja A. Constitutive activation of the Wnt/betacatenin signalling pathway in acute myeloid leukaemia. Oncogene. 2005;24(14):24102420.

5. Ysebaert L, Chicanne G, Demur C, et al. Expression of beta-catenin by acute myeloid leukemia cells predicts enhanced clonogenic capacities and poor prognosis. Leukemia. 2006;20(7):1211-1216.

6. Wang Y, Krivtsov AV, Sinha AU, et al. The Wnt/beta-catenin pathway is required for the development of leukemia stem cells in AML. Science. 2010;327(5973):1650-1653.

7. Zhao C, Blum J, Chen A, et al. Loss of betacatenin impairs the renewal of normal and CML stem cells in vivo. Cancer Cell. 2007; 12(6):528-541.

8. Muller-Tidow C, Steffen B, Cauvet T, et al. Translocation products in acute myeloid leukemia activate the Wnt signaling pathway in hematopoietic cells. Mol Cell Biol. 2004;24(7):2890-2904.

9. Coluccia AM, Vacca A, Dunach M, et al. Bcr$\mathrm{Abl}$ stabilizes beta-catenin in chronic myeloid leukemia through its tyrosine phosphorylation. EMBO J. 2007;26(5):1456-1466.

10. Griffiths EA, Golding MC, Srivastava P, et al. Pharmacological targeting of beta-catenin in normal karyotype acute myeloid leukemia blasts. Haematologica. 2015;100(2):e49-52.

11. Morgan RG, Pearn L, Liddiard K, et al. gamma-Catenin is overexpressed in acute myeloid leukemia and promotes the stabilization and nuclear localization of betacatenin. Leukemia. 2013;27(2):336-343.

12. Luis TC, Ichii M, Brugman MH, Kincade $P$, Staal FJ. Wnt signaling strength regulates normal hematopoiesis and its deregulation is involved in leukemia development. Leukemia. 2012;26(3):414-421.

13. Morgan RG, Mortensson E, Legge DN, et al. LGR5 expression is regulated by EGF in early colorectal adenomas and governs EGFR inhibitor sensitivity. Br J Cancer. 2018;118(4):558-565.

14. Morey L, Brenner C, Fazi F, et al. MBD3, a component of the NuRD complex, facilitates chromatin alteration and deposition of epigenetic marks. Mol Cell Biol. 2008; 28(19):5912-5923.

15. Boukarabila H, Saurin AJ, Batsche E, et al.
The PRC1 Polycomb group complex interacts with PLZF/RARA to mediate leukemic transformation. Genes Dev. 2009;23(10): 1195-1206

16. Kim JJ, Khalid O, Vo S, Sun HH, Wong DT, Kim Y. A novel regulatory factor recruits the nucleosome remodeling complex to wingless integrated (Wnt) signaling gene promoters in mouse embryonic stem cells. J Bio Chem. 2012;287(49):41103-41117.

17. Chiacchiera F, Rossi A, Jammula S, et al. Polycomb Complex PRC1 Preserves Intestinal Stem Cell Identity by Sustaining Wnt/beta-Catenin Transcriptional Activity. Cell Stem Cell. 2016;18(1):91-103.

18. Byers RJ, Currie T, Tholouli E, Rodig SJ, Kutok JL. MSI2 protein expression predicts unfavorable outcome in acute myeloid leukemia. Blood. 2011;118(10):2857-2867.

19. Kaeda J, Ringel F, Oberender C, et al. Up-regulated MSI2 is associated with more aggressive chronic myeloid leukemia. Leuk Lymphoma. 2015;56(7):2105-2113.

20. Li Z, Jin H, Mao G, Wu L, Guo O. Msi2 plays a carcinogenic role in esophageal squamous cell carcinoma via regulation of the Wnt/betacatenin and Hedgehog signaling pathways. Exp Cell Res. 2017;361(1):170-177.

21. Helsmoortel HH, De Moerloose B, Pieters T, et al. LIN28B is over-expressed in specific subtypes of pediatric leukemia and regulates IncRNA H19. Haematologica. 2016;101(6) e240-244

22. Zhou J, Bi C, Ching YQ, et al. Inhibition of LIN28B impairs leukemia cell growth and metabolism in acute myeloid leukemia. J Hematol Oncol. 2017;10(1):138.

23. Tu HC, Schwitalla S, Qian Z, et al. LIN28 cooperates with WNT signaling to drive invasive intestinal and colorectal adenocarcinoma in mice and humans. Genes Dev. 2015;29(10):1074-1086.

24. Yassin ER, Abdul-Nabi AM, Takeda A, Yaseen NR. Effects of the NUP98-DDX10 oncogene on primary human CD34+ cells: role of a conserved helicase motif. Leukemia. 2010;24(5):1001-1011.

25. Gu TL, Mercher T, Tyner JW, et al. A novel fusion of RBM6 to CSF1R in acute megakaryoblastic leukemia. Blood. 2007; 110(1):323-333

26. Ma Z, Morris SW, Valentine V, et al. Fusion of two novel genes, RBM15 and MKL1, in the $t(1 ; 22)(p 13 ; q 13)$ of acute megakaryoblastic leukemia. Nat Genet. 2001;28(3):220-221.

27. Wang Y XW, Wei SM, Chen XM, Wei L, Tian RH, Meng Li, Xiao BR, Wu PX, Yu YH. Highly expressed DDX10 promotes hepatocellular carcinoma cell proliferation through $W n t / \beta$-catenin signaling. Int J Clin Exp Pathol. 2017;10(5):6047-6053.

28. Niu C, Zhang J, Breslin P, Onciu M, Ma Z, Morris SW. c-Myc is a target of RNA-bind ing motif protein 15 in the regulation of adult hematopoietic stem cell and megakaryocyte development. Blood.
2009;114(10):2087-2096

29. Naudin C, Hattabi A, Michelet F, et al PUMILIO/FOXP1 signaling drives expansion of hematopoietic stem/progenitor and leukemia cells. Blood. 2017;129(18):24932506.

30. Lee KY, Chan KY, Tsang KS, et al Ubiquitous expression of MAKORIN-2 in normal and malignant hematopoietic cells and its growth promoting activity. PLoS One. 2014:9(3):e92706.

31. Yang L, Han Y, Suarez Saiz F, Minden MD. A tumor suppressor and oncogene: the WT1 story. Leukemia. 2007;21(5):868-876.

32. Jamieson C, Sharma M, Henderson BR Regulation of beta-catenin nuclear dynamics by GSK-3beta involves a LEF-1 positive feedback loop. Traffic. 2011;12(8):983-999.

33. Simcha I, Shtutman M, Salomon D, et al. Differential nuclear translocation and transactivation potential of beta-catenin and plakoglobin. J Cell Biol. 1998;141(6):14331448.

34. Krieghoff E, Behrens J, Mayr B. Nucleo-cytoplasmic distribution of beta-catenin is regulated by retention. J Cell Sci. 2006;119(Pt 7):1453-1463

35. Behrens J, von Kries JP, Kuhl M, et al Functional interaction of beta-catenin with the transcription factor LEF-1. Nature. 1996; 382(6592):638-642.

36. Davies ML, Roberts GT, Stuart N, Wakeman JA. Analysis of a panel of antibodies to APC reveals consistent activity towards an unidentified protein. Br J Cancer. 2007; 97(3):384-390.

37. Daud SS. The Role of WNT Transcription Factor TCF7L2 in Acute Myeloid Leukaemia. Cardiff University. 2014. Available from: http:// orca.cf.ac.uk/id/eprint/57554 [Last accessed Jan 2019]

38. Petropoulos K, Arseni N, Schessl C, et al. A novel role for Lef-1, a central transcription mediator of Wnt signaling, in leukemogenesis. J Exp Med. 2008;205(3):515-522.

39. Hovanes K, Li TW, Munguia JE, et al. Betacatenin-sensitive isoforms of lymphoid enhancer factor-1 are selectively expressed in colon cancer. Nat Genet. 2001;28(1):53 57.

40. Koch U, Wilson A, Cobas M, Kemler R, Macdonald HR, Radtke F. Simultaneous loss of beta- and gamma-catenin does not perturb hematopoiesis or lymphopoiesis. Blood. 2008;111(1):160-164

41. Trompouki E, Bowman TV, Lawton LN, et al. Lineage regulators direct BMP and Wnt pathways to cell-specific programs during differentiation and regeneration. Cell. 2011;147(3):577-589.

42. Prieve MG, Guttridge KL, Munguia JE, Waterman ML. The nuclear localization signal of lymphoid enhancer factor-1 is recognized by two differentially expressed Srp1nuclear localization sequence receptor pro- 
teins. J Biol Chem. 1996;271(13):7654-7658

43. Hendriksen J, Fagotto F, van d, V, van SM, Noordermeer J, Fornerod M. RanBP3 enhances nuclear export of active (beta)catenin independently of CRM1. J Cell Biol. 2005;171(5):785-797.

44. Griffin JN, Del Viso F, Duncan AR, et al. RAPGEF5 Regulates Nuclear Translocation of beta-Catenin. Dev Cell. 2018;44(2):248260.e4

45. Lu Y, Xie S, Zhang W, et al. Twa1/Gid8 is a beta-catenin nuclear retention factor in Wnt signaling and colorectal tumorigenesis. Cell Res. 2017;27(12):1422-1440.

46. Skokowa J, Cario G, Uenalan M, et al. LEF1 is crucial for neutrophil granulocytopoiesis and its expression is severely reduced in congenital neutropenia. Nat Med. 2006;12(10): 1191-1197.

47. Stoddart A, Wang J, Hu C, et al. Inhibition of WNT signaling in the bone marrow niche prevents the development of MDS in the Apc(del/+) MDS mouse model. Blood. 2017; 129(22):2959-2970
48. Li L, Sheng Y, Li W, et al. beta-Catenin Is a Candidate Therapeutic Target for Myeloid Neoplasms with del(5q). Cancer Res. 2017; 77(15):4116-4126.

49. Jiang X, Mak PY, Mu H, et al. Disruption of Wnt/beta-Catenin Exerts Antileukemia Activity and Synergizes with FLT3 Inhibition in FLT3-Mutant Acute Myeloid Leukemia. Clin Cancer Res. 2018;24(10): 2417-2429.

50. Dandekar S, Romanos-Sirakis E, Pais F, et al. Wnt inhibition leads to improved chemosensitivity in paediatric acute lymphoblastic leukaemia. Br J Haematol. 2014:167(1):87-99.

51. Minke KS, Staib P, Puetter A, et al. Small molecule inhibitors of WNT signaling effectively induce apoptosis in acute myeloid leukemia cells. Eur J Haematol. 2009;82(3): 165-175

52. Gandhirajan RK, Staib PA, Minke K, et al Small molecule inhibitors of Wnt/betacatenin/lef-1 signaling induces apoptosis in chronic lymphocytic leukemia cells in vitro and in vivo. Neoplasia. 2010;12(4):326-335.

53. Hovanes K, Li TW, Waterman ML. The human LEF-1 gene contains a promoter preferentially active in lymphocytes and encodes multiple isoforms derived from alternative splicing. Nucleic Acids Res. 2000; 28(9):1994-2003.

54. Shy BR, Wu CI, Khramtsova GF, et al Regulation of Tcf7l1 DNA binding and protein stability as principal mechanisms of Wnt/beta-catenin signaling. Cell Rep. 2013; 4(1):1-9.

55. Yamada M, Ohnishi J, Ohkawara B, et al. NARF, an nemo-like kinase (NLK)-associated ring finger protein regulates the ubiquitylation and degradation of $\mathrm{T}$ cell factor/lymphoid enhancer factor (TCF/LEF). J Bio Chem. 2006:281(30):20749-20760

56. Gupta K, Kuznetsova I, Klimenkova O, et al. Bortezomib inhibits STAT5-dependent degradation of LEF-1, inducing granulocytic differentiation in congenital neutropenia CD34(+) cells. Blood. 2014;123(16):25502561 